\title{
一种基于 RBFNN 的变体飞机高精度 自适应反步控制方法
}

\author{
谯富祥，史静平，章卫国，吕永筀，屈晓波
}

(西北工业大学 自动化学院, 陕西 西安 710072)

\begin{abstract}
摘 要: 针对变体飞机非线性模型的不确定性问题,提出了一种基于径向基神经网络 (radial basis function neural networks, RBFNN) 的高精度自适应反步控制方法。首先,在变体飞机静态和动态气动 参数分析的基础上,运用传统反步法设计了非线性控制律,并引入径向基神经网络在线逼近系统的不 确定项,提高系统鲁棒性; 并设计鲁棒项消除径向基神经网络带来的逼近误差。其次,通过对虚拟控 制变量进行求导项设计微分跟踪器,解决了传统反步法中存在的 “微分膨胀” 问题。通过 Lyapunov 稳 定性分析,证明该方法能保证闭环系统跟踪误差最终收敛且一致有界。最后,基于 Matlab/Simulink 搭 建了变体飞机的数字仿真模型, 并与常规反步法进行了对比分析,仿真结果表明该方法具有控制精度 高、鲁棒性强的特点。
\end{abstract}

\section{关 键 词: 变体飞机; 反步法;径向基网络;自适应控制}

中图分类号: V249.1

文献标志码: A

变体飞机能改变其气动外形,使其在执行不同 飞行任务时都能保证飞行性能最优 ${ }^{[1]}$ 。由于变体 飞机优良的飞行性能和适应能力,吸引了越来越多 的学者对其进行研究。对于机翼变化的变体飞机， 机翼的过渡过程十分复杂而且重要。此类变体飞机 在变形过程中, 由于质量分布、气动力和力矩的显著 变化,形成了具有内外不确定性的复杂时变非线性 动力学问题, 变体飞机的这些不确定和时变的特性 给控制系统的设计带来了困难 ${ }^{[2-4]}$ 。

变体飞机属于跨学科研究, 包含有机翼结构、驱 动系统、气动建模、非刚性动力学和飞行控制等领 域 ${ }^{[5]}$ 。一部分研究主要集中在变形结构的执行机 构位置优化上面 ${ }^{[6-8]}$ 。还有其他相关的研究工作体 现在气动弹性分析方面 ${ }^{[9]}$, 以及为减少飞行控制中 必须处理的动力学问题, 对变体飞机的惯性力和力 矩展开研究 ${ }^{[10]}$ 。文献 $[11]$ 提出了一种适用于变体 飞机动态载荷数值计算的方法。在文献 [12] 中, 提 出了折叠翼型飞机在机翼变形过程中的线性参数变 化模型, 并基于准稳态气动假设对纵向动力响应进
文章编号 : 1000-2758(2020)03-0540-10

行了数值模拟。

尽管机翼结构、驱动系统和动力学模型的发展 取得了重大进展,但要有效地控制变体飞机仍有许 多工作要做。在变体飞机几何形状发生较大变化 时,气动力、力矩和质心会受到严重影响。因此变体 飞机的控制系统必须能够在变形情况下,保持整个 飞行系统的稳定, 要具备良好的鲁棒性。为此, 国内 外学者都开展了相关的研究。文献 [13]引人了线 性输人变化来描述此类系统,并应用合成的一对线 性控制器去解决线性输人变体飞行器的干扰抑制问 题。文献 $[14]$ 针对变翼展变体飞机进行了纵向飞 行稳定性和控制的研究, 并提出了一种简单的比例 状态反馈控制与特征结构分配相结合的方法。针对 无尾折翼变体飞机的线性模型, 文献 [15]设计了多 回路的飞机变形控制器, 并提出了一种自增益 $H_{\infty}$ 控制方法 ( self-gain scheduled $H_{\infty}$ controller) 保证机 翼过渡过程的稳定性和动态性。为了保证变体无人 机在几何外形大尺度变化下仍具有良好性能, 文献 [16]在变线性参数和经典方法的基础上,设计了一 
种多回路控制器,利用经典的控制方法通过内环增 益来保证系统的稳定性, 同时设计了外环变参数控 制器来保证控制器的动态性能。

而对于变体飞机系统中的不确定性, 大多学者 运用自适应控制和神经网络控制技术进行解决, 这 些技术已经发展了几十年, 广泛应用于解决系统的 不确定性问题。文献 [17] 设计了一种基于滚动时 域优化和改进的序列最小二乘参数辨识的间接自适 应控制方法, 用于解决线性变体飞机动力学模型中 的不确定性。文献 [18]针对变体飞行器的控制设 计了一种单网络自适应跟踪控制器, 不过需要采用 线性系统模型离线预训练神经网络的初始权值。文 献 [19] 基于反馈线性化的思想, 针对变体飞行器提 出了一种将动态逆方法与结构模型参考自适应控制 相结合的控制方法。由于变体飞机的外形设计和飞 行条件的变化, 它对物理和气动参数的变化极为敏 感,典型的变体飞机表现出高度非线性动力学特性。 但几乎上述控制器的设计都是基于线性模型的。

反步法作为一种强大的非线性技术, 利用其设 计的控制器已经广泛应用于具有严格反馈形式的系 统中。但对于传统的反步法而言, 需要面对系统建 模的不确定性引起的问题, 其中之一便是非线性项 微分所带来的 “微分膨胀” 问题。为了有效处理各 子系统的系统不确定性, 文献[20-21] 中采用了具有 全局逼近能力的 RBFNN。 RBFNN 作为一种单隐层 神经网络, 学习收玫速度快, 具有优良的逼近能 力 $^{[22]}$ 。 RBFNN 是具有唯一最佳逼近( 克服局部极 小值问题)、训练简洁、学习收敛速度快等良好性能 的前馈型神经网络, 目前已证明 RBFNN 能够以任 意精度逼近任意连续的非线性网络, 被广泛用于函 数逼近、语音识别、模式识别、图像处理、自动控制和 故障诊断等领域。将 RBFNN 运用到反步法设计的 非线性控制器中, 用其去处理各子系统中的系统不 确定性, 这种针对变体飞机的控制器设计思想将能 有效地处理变体飞机模型不准确带来的问题。

本文针对一款变后掠翼无人飞机设计了基于 RBFNN 的高精度自适应反步控制器。引人微分跟 踪器 (tracking differentiator, TD $)^{[23]}$ 来改善传统反步 法中所存在的 “微分膨胀” 问题。设计了鲁棒项消 除了 RBFNN 带来的逼近误差, 并利用 Lyapunov 稳 定性分析方法证明了所设计的控制律能够保证闭环 系统跟踪误差收敛且一致有界。通过仿真验证了该 方法的优越性。

\section{1 问题描述}

本文所针对的研究对象是一款变后掠翼无人飞 机,如图 1 所示。

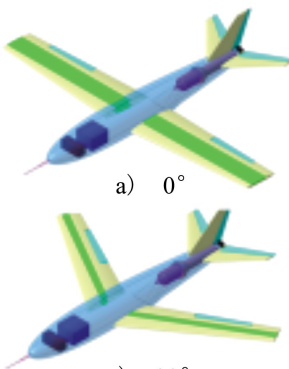

c) $30^{\circ}$

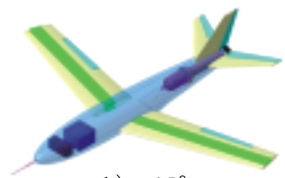

b) $15^{\circ}$

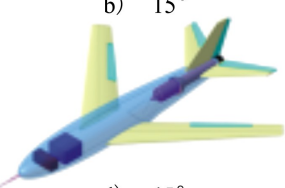

d) $45^{\circ}$
图 1 不同构型下的变体飞机

该变体飞机推力方向沿发动机轴线且与机身轴 线重合, 变体飞行器纵向模型的非线性方程组可以 按照其受力情况得到如下数学描述

$$
\begin{gathered}
\dot{V}=\frac{1}{m}\left(-D_{w}+T \cos \alpha-m g \sin \gamma\right) \\
\dot{h}=V \sin \gamma \\
\dot{\gamma}=\frac{1}{m V}\left(L_{w}+T \sin \alpha-m g \cos \gamma\right) \\
\dot{\alpha}=q-\dot{ } \\
\dot{q}=\frac{1}{I_{y}} M_{b y}
\end{gathered}
$$

式中, 状态量 $\alpha, \gamma, q, V, h$ 分别表示飞行器的迎角、 航迹角、俯仰角速率、飞行速度以及飞行高度; $m, g$ 和 $I_{y}$ 分别表示飞行器的质量、重力加速度和其沿 $y$ 轴的转动惯量; $L_{w}, D_{w}, T$ 和 $M_{b y}$ 分别为飞行器所受到 的升力、阻力、推力以及俯仰力矩。值得注意的是, 气动参数和飞机的其他设计参数都是随后掠角的变 化而变化,具体说明见附录 $\mathrm{I}$ 。

设 $x_{1}=\alpha, x_{2}=q, \boldsymbol{x}=\left[\begin{array}{lllll}\alpha & q & V & \gamma & h\end{array}\right]^{\mathrm{T}}$, $u=\delta_{e}$, 可将变体飞行器模型改写为下面的形式

$$
\left\{\begin{array}{l}
\dot{x}_{1}=f_{1}(\boldsymbol{x})+g_{1}(\boldsymbol{x}) x_{2}+\Delta_{1} \\
\dot{x_{2}}=f_{2}(\boldsymbol{x})+g_{2}(\boldsymbol{x}) u+\Delta_{2} \\
y=x_{1}
\end{array}\right.
$$

式中, $\Delta_{1}, \Delta_{2}$ 为系统的不确定项, 主要包括建模误差 $\Delta f_{1}(\boldsymbol{x}), \Delta f_{2}(\boldsymbol{x}), \Delta g_{1}(\boldsymbol{x}), \Delta g_{2}(\boldsymbol{x})$ 和外界干扰 $d_{1}, d_{2}$

$$
\left\{\begin{array}{l}
\Delta_{1}=\Delta f_{1}(\boldsymbol{x})+\Delta g_{1}(\boldsymbol{x}) x_{2}+d_{1} \\
\Delta_{2}=\Delta f_{2}(\boldsymbol{x})+\Delta g_{2}(\boldsymbol{x}) u+d_{2}
\end{array}\right.
$$

设计控制律使得系统输出 $y$ 可以稳定跟踪输人 信号 $y_{d}$, 主要是能够消除不确定项对系统的影响。 


\section{2 自适应控制律设计}

\section{1 相关假设}

先给出一些与系统控制律设计相关的假设:

(1)给定的有界参考信号 $y_{d}$ 连续可微且一阶导 数有界;

(2) $g_{1}(\boldsymbol{x})$ 和 $g_{2}(\boldsymbol{x})$ 可逆;

(3) 系统的不确定项 $\Delta_{1}, \Delta_{2}$ 有界;

(4) 给定任意逼近误差上界 $\varepsilon_{M}$, 存在理想的权值 $\boldsymbol{w}$, 该权值有上界 $w_{M}$, 使得 RBFNN 一致逼近系统中 连续光滑未知的 $\Delta$, 即

$$
\left\{\begin{array}{l}
\boldsymbol{w}^{\mathrm{T}} \boldsymbol{v}=\Delta-\varepsilon,|\varepsilon| \leqslant \varepsilon_{M},|\boldsymbol{w}| \leqslant w_{M} \\
\boldsymbol{w}=\hat{\boldsymbol{w}}+\widetilde{\boldsymbol{w}}
\end{array}\right.
$$

式中: $\varepsilon$ 表示 RBFNN 的逼近误差; $\hat{\boldsymbol{w}}$ 为 RBFNN 的实 际权值; $\widetilde{\boldsymbol{w}}$ 为理想权值与实际权值之差。

\section{2 微分跟踪器设计}

在使用反步法进行设计控制律时, 需要对设计 出的虚拟控制变量进行求导, 而由于模型误差和系 统噪声, 使用传统微分器进行求导不可避免的会出 现 “微分膨胀” 的问题, 而利用微分跟踪器来估计虚 拟控制变量的一阶导数可以有效避免此类问题。

引理 1 若输人信号 $r(t)$ 分段连续可导, 且输 人信号的前 $n-2$ 阶导数均存在, 允许在某时刻 $t_{j}$ $(j=1,2, \cdots k)$, 输人信号的 $n-1$ 阶导数不可导, 但 其左导数 $r_{-}^{(n-1)}\left(t_{j}\right)$ 与右导数 $r_{+}^{(n-1)}\left(t_{j}\right)$ 均存在( 同时 $\left.r_{-}^{(n-1)}\left(t_{j}\right) \neq r_{+}^{(n-1)}\left(t_{j}\right)\right)$, 则存在如下的微分跟踪器 ( TD )

$$
\left\{\begin{array}{l}
\zeta_{1}=\zeta_{2} \\
\zeta_{2}=\zeta_{3} \\
\zeta_{n}=-K^{n}\left[a_{1} \tanh \left(\zeta_{1}-r\right)+a_{2} \tanh \left(\frac{\zeta_{2}}{K}\right)+\right. \\
\left.\quad a_{n} \tanh \left(\frac{\zeta_{n}}{K^{n-1}}\right)\right]
\end{array}\right.
$$

式中, $K>0, a_{i}(i=1,2, \cdots, n)>0$ 为待设计参数。 且存在 $\partial>0, l \partial>n$ 使得

$$
\zeta_{i}-r^{i-1}(t)=O\left(\left(\frac{1}{K}\right)^{l \partial-i+1}\right), i=1,2 \cdots n
$$

式中, $O\left((1 / K)^{l \partial-i+1}\right)$ 表示 $\zeta_{i}$ 与 $r^{i-1}(t)$ 之差是 $(1 / K)^{l \partial-i+1}$ 的高阶无穷小, $\partial=\left(1-K_{0}\right) / K_{0}, K_{0} \in$ $[0, \min (l /(l+n), 0.5)], n>2$
由 (9) 式可知, $\zeta_{1}$ 是 $r(t)$ 的估计值, $\zeta_{i}$ 是 $r(t)$ 第 $i-1$ 阶导数的估计值。由 (10) 式可知, 选取较大的 参数 $K$ 可以尽可能减小输人信号导数的估计误差。 反步法只需要虚拟控制变量的一阶导数, 故而选取 的 TD 为

$$
\left\{\begin{array}{l}
\dot{\zeta}_{1}=\zeta_{2} \\
\dot{\zeta}_{2}=-K^{2}\left[a_{1} \tanh \left(\zeta_{1}-r\right)+a_{2} \tanh \left(\frac{\zeta_{2}}{K}\right)\right]
\end{array}\right.
$$

\section{3 控制律设计}

Step1 考虑到系统 (6) 的第一个子系统 $\dot{x}_{1}=$ $f_{1}(x)+g_{1}(x) x_{2}+\Delta_{1}$, 定义虚拟反馈误差

$$
\left\{\begin{array}{l}
e_{1}=x_{1}-y_{d} \\
e_{2}=x_{2}-x_{2 d}
\end{array}\right.
$$

式中, $x_{2 d}$ 为第二个子系统的虚拟控制量。

对于 $e_{1}$ 求导, 得

$$
\dot{e}_{1}=\dot{x}_{1}-\dot{y}_{d}=f_{1}(\boldsymbol{x})+g_{1}(\boldsymbol{x}) x_{2}+\Delta_{1}-\dot{y}_{d}
$$

根据 (8) 式设计的 RBF 在线逼近系统的不确定 项为

$$
\Delta_{1}=\boldsymbol{w}_{1}^{\mathrm{T}} \boldsymbol{v}_{1}+\varepsilon_{1}=\left(\hat{\boldsymbol{w}}_{1}^{\mathrm{T}}+\widetilde{\boldsymbol{w}}_{1}^{\mathrm{T}}\right) \boldsymbol{v}_{1}+\varepsilon_{1}
$$

将 (12)、(14) 式代人(13) 式可得

$$
\begin{gathered}
\dot{e}_{1}=f_{1}(\boldsymbol{x})+g_{1}(\boldsymbol{x}) e_{2}+g_{1}(\boldsymbol{x}) x_{2 d}+ \\
\boldsymbol{w}_{1}^{\mathrm{T}} \boldsymbol{v}_{1}+\boldsymbol{\varepsilon}_{1}-\dot{y}_{d} \\
\text { 令第一个子系统的理想虚拟控制量为 }
\end{gathered}
$$

$$
\begin{aligned}
& x_{2 d}=g_{1}^{-1}(\boldsymbol{x})\left[-f_{1}(\boldsymbol{x})-k_{1} e_{1}-\hat{\boldsymbol{w}}_{1}^{\mathrm{T}} \boldsymbol{v}_{1}-\right. \\
& \left.\sigma_{1} \tanh \left(e_{1}\right)+\dot{y}_{d}\right]
\end{aligned}
$$

式中, $k_{1}, \sigma_{1}$ 都为正实数。 $\hat{\boldsymbol{w}}_{1}^{\mathrm{T}} \boldsymbol{v}_{1}$ 是 $\mathrm{RBFNN}$ 输出, 用 以逼近系统不确定项 $\Delta_{1} ; \sigma_{1} \tanh \left(e_{1}\right)$ 为鲁棒项, 用 以抵消 RBFNN 的逼近误差 $\varepsilon_{1}$ 。

本文所采用的 RBFNN 是一种单隐层神经网 络,学习收玫速度快, 其数学表达式为

$$
\boldsymbol{N}\left(\boldsymbol{w}, \boldsymbol{x}_{n}\right)=\boldsymbol{w}^{\mathrm{T}} \boldsymbol{v}=\boldsymbol{w}^{\mathrm{T}} \boldsymbol{v}\left(\boldsymbol{x}_{n}\right)
$$

式中: $\boldsymbol{N}\left(\boldsymbol{w}, \boldsymbol{x}_{n}\right)=\left[\begin{array}{lll}N_{1}\left(\boldsymbol{x}_{n}\right) & \cdots & N_{p}\left(\boldsymbol{x}_{n}\right)\end{array}\right] \in \mathbf{R}^{p}$ 为 RBFNN 的输出向量; $x_{n} \in \mathbf{R}^{k}$ 为 RBFNN 的输人向 量; $\boldsymbol{w}=\left[\begin{array}{lll}w_{1} & \cdots & w_{p}\end{array}\right] \in \mathbf{R}^{l \times p}$ 为 $\mathrm{RBFNN}$ 的权值; $l$ 为 RBFNN 节点个数; $\boldsymbol{v}\left(\boldsymbol{x}_{n}\right)=\left[\begin{array}{lll}v_{1}\left(x_{n}\right) & \cdots & v_{l}\left(x_{n}\right)\end{array}\right] \in$ $\mathbf{R}^{l}, v_{i}\left(x_{n}\right)$ 为基函数, 通常选择为高斯函数, 有

$$
v_{i}\left(x_{n}\right)=\exp \left(-\frac{\left\|\boldsymbol{x}_{n}-\boldsymbol{c}_{i}\right\|^{2}}{b_{i}^{2}}\right)(i=1, \cdots, l)
$$

式中: $\boldsymbol{c}_{i} \in \mathbf{R}^{k}$ 为高斯基函数中心向量; $b_{i}$ 为高斯基 函数的基宽。 $\mathrm{RBF}$ 属于线性参数化神经网络, 网络 
输出是基函数的线性组合,其结构如图 2 所示。

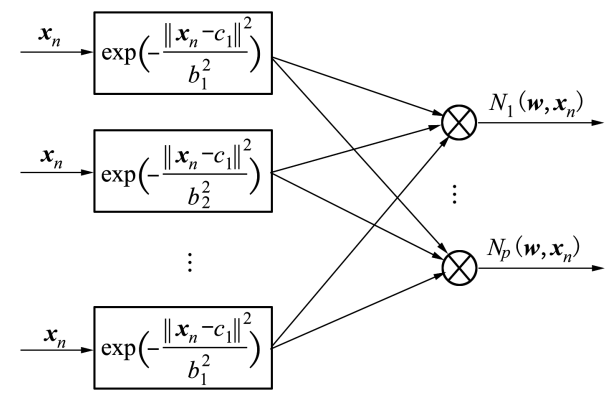

图 2 RBFNN 基本结构

将 (16) 式带人 (15) 式可得

$$
\begin{gathered}
\dot{e}_{1}=-k_{1} e_{1}+g_{1}(\boldsymbol{x}) e_{2}+\widetilde{\boldsymbol{w}}_{1}^{\mathrm{T}} \boldsymbol{v}_{1}+ \\
\varepsilon_{1}-\sigma_{1} \tanh \left(e_{1}\right)
\end{gathered}
$$

式中: 参数 $k_{1}$ 用于调节系统的动态特性; 参数 $\sigma_{1}$ 改 善系统的鲁棒性。本文集中研究鲁棒自适应反步控 制方法, 对参数的选取只是从理论上给予约束条件 和证明,在实际中利用经验和“试凑” 的方法在仿真 中确定这些参数。

Step2 考虑到系统 (6) 的第二个子系统 $\dot{x}_{2}=$ $f_{2}(x)+g_{2}(x) u+\Delta_{2}$, 对 $e_{2}$ 求导可得

$$
\begin{aligned}
& \dot{e_{2}}=\dot{x}_{2}-\dot{x}_{2 d}= \\
& f_{2}(x)+g_{2}(x) u+\Delta_{2}-\dot{x}_{2 d}
\end{aligned}
$$

根据 (8) 式, 设计 RBFNN 在线逼近系统的不确 定项

$$
\Delta_{2}=\boldsymbol{w}_{2}^{\mathrm{T}} \boldsymbol{v}_{2}+\boldsymbol{\varepsilon}_{2}=\left(\hat{\boldsymbol{w}}_{2}^{\mathrm{T}}+\widetilde{\boldsymbol{w}}_{2}^{\mathrm{T}}\right) \boldsymbol{v}_{2}+\boldsymbol{\varepsilon}_{2}
$$

与 $x_{2 d}$ 的设计类似, 取 $u_{d}$ 即 $u$

$$
\begin{gathered}
u_{d}=g_{2}^{-1}(\boldsymbol{x})\left[-f_{2}(\boldsymbol{x})-g_{1}(\boldsymbol{x}) e_{1}-k_{2} e_{2}-\right. \\
\left.\hat{\boldsymbol{w}}_{2}^{\mathrm{T}} \boldsymbol{v}_{2}-\sigma_{2} \tanh \left(e_{2}\right)+\dot{x}_{2 d}\right]
\end{gathered}
$$

式中, $k_{2}, \sigma_{2}$ 都为正实数。 $\hat{\boldsymbol{w}}_{2}^{\mathrm{T}} \boldsymbol{v}_{2}$ 是 $\mathrm{RBFNN}$ 输出, 用 以逼近系统不确定项 $\Delta_{2} ; \sigma_{2} \tanh \left(e_{2}\right)$ 为鲁棒项, 用 以抵消 RBFNN 的逼近误差 $\varepsilon_{2}$ 。

将(22) 式带人(20) 式可得

$$
\begin{gathered}
\dot{e}_{2}=-k_{2} e_{2}-g_{1}(\boldsymbol{x}) e_{1}+\widetilde{\boldsymbol{w}}_{2}^{\mathrm{T}} \boldsymbol{v}_{2}+ \\
\varepsilon_{2}-\sigma_{2} \tanh \left(e_{2}\right)
\end{gathered}
$$

\section{4 稳定性分析}

取 Lyapunov 函数

$$
L_{V}=\frac{1}{2} e_{1}^{2}+\frac{1}{2 \lambda_{1}} \operatorname{tr}\left(\widetilde{\boldsymbol{w}}_{1}^{\mathrm{T}} \widetilde{\boldsymbol{w}}_{1}\right)+\frac{1}{2} e_{2}^{2}+\frac{1}{2 \lambda_{2}} \operatorname{tr}\left(\widetilde{\boldsymbol{w}}_{2}^{\mathrm{T}} \widetilde{\boldsymbol{w}}_{2}\right)
$$

\section{对其求导得}

$$
\dot{L}_{V}=e_{1} \dot{e}_{1}+\frac{1}{\lambda_{1}} \operatorname{tr}\left(\widetilde{\boldsymbol{w}}_{1}^{\mathrm{T}} \widetilde{\boldsymbol{w}}_{1}\right)+e_{2} \dot{e}_{2}+\frac{1}{\lambda_{2}} \operatorname{tr}\left(\widetilde{\boldsymbol{w}}_{2}^{\mathrm{T}} \widetilde{\boldsymbol{w}}_{2}\right)
$$

将(19)式和(23) 式代人(25) 式, 整理可得

$$
\begin{gathered}
\dot{L}_{V}=-k_{1} e_{1}^{2}+e_{1} \widetilde{\boldsymbol{w}}_{1}^{\mathrm{T}} \boldsymbol{v}_{1}-\frac{1}{\lambda_{1}} \sum_{i=1}^{p} \widetilde{w}_{1 i} \hat{w}_{1 i}+ \\
e_{1}\left[\boldsymbol{\varepsilon}_{1}-\sigma_{1} \tanh \left(e_{1}\right)\right]- \\
k_{2} e_{2}^{2}+e_{2} \widetilde{\boldsymbol{w}}_{2}^{\mathrm{T}} \boldsymbol{v}_{2}-\frac{1}{\lambda_{2}} \sum_{i=1}^{p} \widetilde{w}_{2 i} \hat{w}_{2 i}+ \\
e_{2}\left[\varepsilon_{2}-\sigma_{2} \tanh \left(e_{2}\right)\right]
\end{gathered}
$$

设计权值自适应律为 $\left\{\begin{array}{l}\hat{w}_{1 i}=\lambda_{1}\left(e_{1} v_{1 i}-\eta_{1} \hat{w}_{1 i}\right) \\ \hat{w}_{2 i}=\lambda_{2}\left(e_{2} v_{2 i}-\eta_{2} \hat{w}_{2 i}\right)\end{array}\right.$,

$$
\text { 且上式有 }\left\{\begin{array}{l}
e_{1} \widetilde{\boldsymbol{w}}_{1}^{\mathrm{T}} \boldsymbol{v}_{1}=\sum_{i=1}^{p} \widetilde{w}_{1 i} e_{1} v_{1 i} \\
e_{2} \widetilde{\boldsymbol{w}}_{2}^{\mathrm{T}} \boldsymbol{v}_{2}=\sum_{i=1}^{p} \widetilde{w}_{2 i} e_{2} v_{2 i}
\end{array}\right. \text {, 那么 (26) 式可整理 }
$$
为

$$
\begin{aligned}
& \dot{L}_{V}=-k_{1} e_{1}^{2}-k_{2} e_{2}^{2}+e_{1}\left[\varepsilon_{1}-\sigma_{1} \tanh \left(e_{1}\right)\right]+ \\
& e_{2}\left[\varepsilon_{2}-\sigma_{2} \tanh \left(e_{2}\right)\right]+ \\
& \eta_{1} \sum_{i=1}^{p} \tilde{w}_{1 i} \hat{w}_{1 i}+\eta_{2} \sum_{i=1}^{p} \tilde{w}_{2 i} \hat{w}_{2 i}
\end{aligned}
$$

由于

$$
\left\{\begin{array}{c}
\sum_{i=1}^{p} \widetilde{w}_{1 i} \hat{w}_{1 i} \leqslant \frac{1}{2} \sum_{i=1}^{p}\left(w_{1 i}^{2}-\widetilde{w}_{1 i}^{2}\right)= \\
\frac{1}{2}\left|w_{1}\right|^{2}-\frac{1}{2}\left|\widetilde{w}_{1}\right|^{2} \\
\sum_{i=1}^{p} \widetilde{w}_{2 i} \hat{w}_{2 i} \leqslant \frac{1}{2} \sum_{i=1}^{p}\left(w_{2 i}^{2}-\widetilde{w}_{2 i}^{2}\right)= \\
\frac{1}{2}\left|w_{2}\right|^{2}-\frac{1}{2}\left|\tilde{w}_{2}\right|^{2}
\end{array}\right.
$$

故可得到

$$
\begin{aligned}
\dot{L}_{V} & \leqslant\left(\frac{1}{2} \eta_{1}\left|w_{1}\right|^{2}-k_{1} e_{1}^{2}\right)-\frac{1}{2} \eta_{1}\left|\tilde{w}_{1}\right|^{2}+ \\
& e_{1}\left[\varepsilon_{1}-\sigma_{1} \tanh \left(e_{1}\right)\right]+ \\
& \left(\frac{1}{2} \eta_{2}\left|w_{2}\right|^{2}-k_{2} e_{2}^{2}\right)-\frac{1}{2} \eta_{2}\left|\tilde{w}_{2}\right|^{2}+ \\
& e_{2}\left[\varepsilon_{2}-\sigma_{2} \tanh \left(e_{2}\right)\right]
\end{aligned}
$$

设 $w_{1}$ 和 $w_{2}$ 有上界, 满足 $\left|w_{1}\right|<w_{1 M},\left|w_{2}\right|<$ $w_{2 M} ; \varepsilon_{1}, \varepsilon_{2}$ 有上界 $\left|\varepsilon_{1}\right|<\varepsilon_{1 M},\left|\varepsilon_{2}\right|<\varepsilon_{2 M}$; 反馈误 差 $e_{1}, e_{2}$ 有下界, $\left|e_{1}\right| \geqslant e_{1 m} \geqslant 0,\left|e_{2}\right| \geqslant e_{2 m} \geqslant 0$ 。选 择合适的参数 $k_{1}, k_{2}, \eta_{1}, \eta_{2}, \sigma_{1}$ 和 $\sigma_{2}$ 满足 (30) 式, 可确保 $\dot{L}_{V}<0$, 即所设计的控制律能够保证闭环系 统跟踪误差收敛且一致有界。 


$$
\left\{\begin{array}{l}
\frac{k_{1}}{\eta_{1}}>\frac{w_{1 M}^{2}}{2 e_{1 m}^{2}} \\
\frac{k_{2}}{\eta_{2}}>\frac{w_{2 M}^{2}}{2 e_{2 m}^{2}} \\
\sigma_{1}>\frac{\varepsilon_{1 M}}{\tanh \left(e_{1 m}\right)} \\
\sigma_{2}>\frac{\varepsilon_{2 M}}{\tanh \left(e_{2 m}\right)}
\end{array}\right.
$$

\section{3 仿真验证}

\section{1 微分跟踪器仿真验证}

本节将针对本文 2.2 节所引人的微分跟踪器进 行仿真验证。

选择幅值为 10 , 频率 $1 \mathrm{rad} / \mathrm{s}$ 的正弦波信号为 输人信号, 选择噪声功率为 0.1 的白噪声作为噪声 源, 进行仿真。经计算信号及噪声参数如表 1 所示。

\section{表 1 信号数据}

\begin{tabular}{cccc}
\hline 项目 & 信号功率 & 噪声功率 & 信噪比 $/ \mathrm{dB}$ \\
\hline 数值 & 48.39 & 0.11 & 26.28 \\
\hline
\end{tabular}

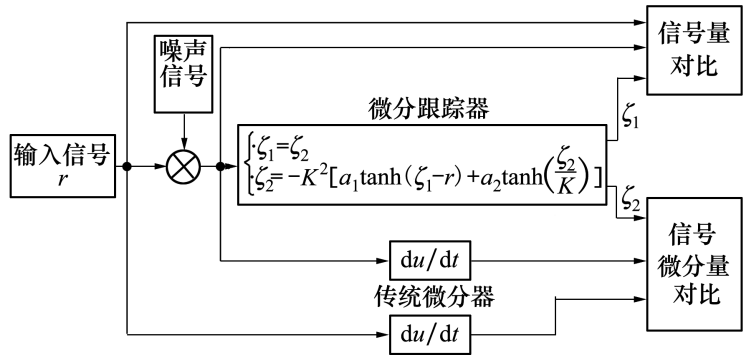

图 3 仿真结构图

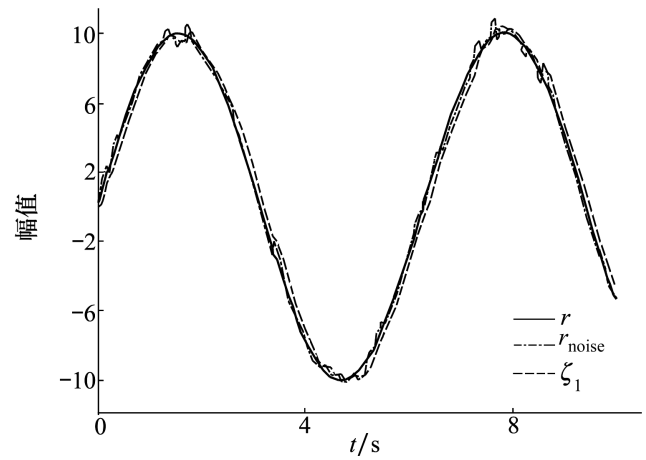

图 4 微分跟踪器原始信号量跟踪对比曲线

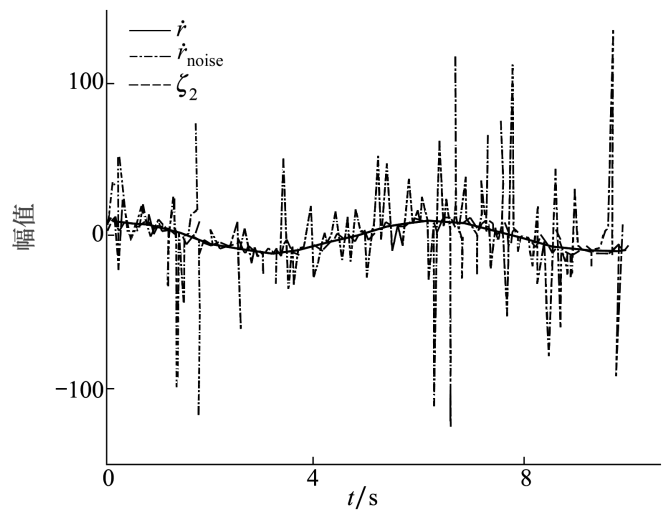

图 5 微分跟踪器与传统微分器对比曲线

\section{2 角速率环仿真验证}

为了验证本文提出方法的优越性,针对变体飞 机纵向模态进行仿真分析。根据控制律设计的规 律, 先对内环 (角速率环) 进行仿真验证, 对比分析 效果, 接着再对外环 (姿态环) 进行仿真验证。

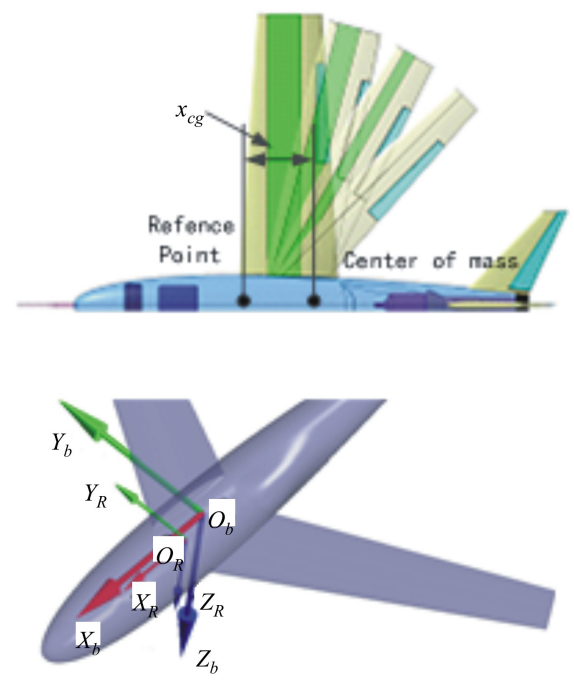

图 6 修正参数 $x_{c g}$ 与参考系定义

本文所研究的变体飞机质量为 $38 \mathrm{~kg}$, 其他性能 参数如表 3 所示。所设计的神经网络包含 50 个 $N\left(w, x_{n}\right)$ 节点, 其中针对内环的输人向量设置为 $\boldsymbol{x}_{n}^{\mathrm{inner}}=\left[\begin{array}{ll}q & \dot{q}\end{array}\right]$, 则取高斯基函数中心向量为 $\boldsymbol{c}_{i}^{\mathrm{inner}}=$ $\left[\begin{array}{ll}q_{\mathrm{cmd}} & \dot{q}_{\mathrm{cmd}}\end{array}\right]$; 针对外环的输人向量设置为 $\boldsymbol{x}_{n}^{\text {outer }}=$ $\left[\begin{array}{ccc}\alpha & \alpha & q\end{array}\right]$, 则取高斯基函数中心向量为 $\boldsymbol{c}_{i}^{\text {outer }}=$ $\left[\begin{array}{lll}\alpha_{\text {cmd }} & \dot{\alpha}_{\mathrm{cmd}} & 0\end{array}\right]$ 。鲁棒自适应反步法控制器和微 分跟踪器中的参数如表 4 所示。 
表 3 不同构型下变体飞机参数

\begin{tabular}{cccccc}
\hline$\tau /\left({ }^{\circ}\right)$ & 0 & 5 & 10 & 15 & 20 \\
$b / \mathrm{m}$ & 3.347 & 3.344 & 3.318 & 3.269 & 3.195 \\
$S_{w} / \mathrm{m}^{2}$ & 1.6 & 1.532 & 1.463 & 1.391 & 1.317 \\
$c_{A} / \mathrm{m}$ & 0.486 & 0.467 & 0.451 & 0.437 & 0.425 \\
$x_{c g} / \mathrm{m}$ & 0.147 & 0.197 & 0.252 & 0.307 & 0.36 \\
$I_{y} /\left(\mathrm{kg} \cdot \mathrm{m}^{-1}\right)$ & 6.490 & 6.592 & 6.765 & 6.998 & 7.275 \\
$\tau /\left({ }^{\circ}\right)$ & $25^{\circ}$ & $30^{\circ}$ & $35^{\circ}$ & $40^{\circ}$ & $45^{\circ}$ \\
$b / \mathrm{m}$ & 3.099 & 2.981 & 2.841 & 2.681 & 2.503 \\
$S_{w} / \mathrm{m}^{2}$ & 1.243 & 1.168 & 1.095 & 1.025 & 0.958 \\
$c_{A} / \mathrm{m}$ & 0.416 & 0.411 & 0.408 & 0.411 & 0.418 \\
$x_{c g} / \mathrm{m}$ & 0.41 & 0.456 & 0.497 & 0.516 & 0.539 \\
$I_{y} /\left(\mathrm{kg} \cdot \mathrm{m}^{-1}\right)$ & 7.576 & 7.882 & 8.168 & 8.415 & 8.606 \\
\hline
\end{tabular}

表 4 参数列表

\begin{tabular}{cccccccc}
\hline 参数 & $K$ & $a_{1}$ & $a_{2}$ & $k_{1}$ & $k_{2}$ & $\sigma_{1}$ & $\sigma_{2}$ \\
\hline 数值 & 45 & 0.6 & 2 & 4 & 10 & 9 & 0.5 \\
\hline
\end{tabular}

为了验证控制系统的鲁棒性, 在模型的标称气 动参数上增加了 $5 \%$, 其模型气动参数变化如下

$$
\left\{\begin{array}{l}
C_{\mathrm{D}}=C_{\mathrm{D}_{\text {_ori }}} \cdot(1+5 \%) \\
C_{\mathrm{L}}=C_{\mathrm{L}_{-} \text {ori }} \cdot(1+5 \%) \\
C_{m}=C_{m_{\text {_ori }}} \cdot(1+5 \%)
\end{array}\right.
$$

下文将在变体飞机的固定构型和变构型 2 种情况 下, 对比传统反步法和基于 RBFNN 的鲁棒自适应 变体飞机反步控制方法的仿真效果。

- 固定构型仿真对比

本节在变体飞机固定构型情况, 对 2 种方法设
计的内环控制律进行仿真比较。固定后掠角构型为 $0^{\circ}\left(\tau=0^{\circ}\right)$, 初始飞行状态设为 $h=1000 \mathrm{~m}, V=$ $30 \mathrm{~m} / \mathrm{s}, \gamma=0^{\circ}$ 。给定的参考俯仰角速率指令如图 7 所示, 指令在 $3 \mathrm{~s}$ 后从 $0 \% \mathrm{~s}$ 增加到 $5 \% \mathrm{~s}$ 保持 $3 \mathrm{~s}$ 后 回归到 $0 \% \mathrm{~s}$, 在 $8 \mathrm{~s}$ 时又变成 $-5 \%$ 并维持 $3 \mathrm{~s}$ 后恢 复到 $0 \% \mathrm{~s}$ 。图 7 展示了仿真结果。

\section{表 5 固定构型下俯仰角速率跟踪误差的均方根对比}

\begin{tabular}{ccc}
\hline 方法 & 传统反步法 & 本文方法 \\
\hline 均方根数值 & 0.7303 & 0.6962 \\
\hline
\end{tabular}

通过对比发现, 在内环中使用本文所提出的方 法相较于传统的反步法, 动态响应更快。如表 5 所 示, 对 2 种方法的俯仰角速率跟踪误差求其均方根 (平方平均数), 可发现传统反步法的结果要高出本 文提出方法 $4.8922 \%$, 这个结果可以反映出本文方 法的优越性。

从控制器最终输出结果看, 在 $8.12 \mathrm{~s}$ 时, 2 种方 法的出舵量出现最大偏差, 本文方法计算的升降舵 出舵量高出传统反步法的 $4.68 \%$ 。对比数据可以看 出由于控制指令在第 $6 \mathrm{~s}$ 和第 $8 \mathrm{~s}$ 的剧烈切换, 导致 控制器计算出相应的舵偏输出, 但是传统反步法的 响应相对来说有所迟滞, 动态性能欠佳。

\section{- 变构型仿真对比}

为了进一步测试本文提出方法的性能, 将在给 定控制指令的同时对构型也进行改变。后掠角在仿 真开始 $3 \mathrm{~s}$ 后开始以 $9^{\circ} / \mathrm{s}$ 的速率从变化 $0^{\circ}$ 到 $45^{\circ}$, 通过(32) 式的二阶指令滤波器对其进行指令软化, 其后掠角变化曲线如图 8 所示, 除此之外其他仿真 条件与上节相同。

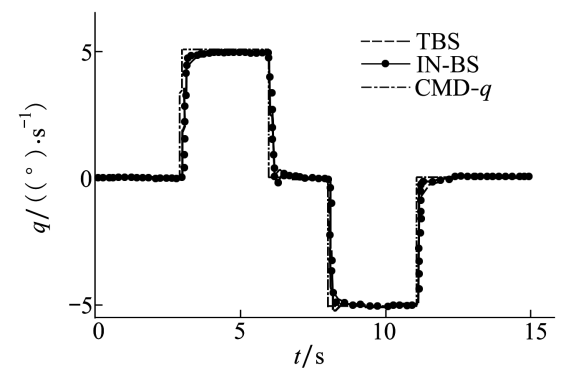

图 7 固定构型下俯仰角速率变化曲线

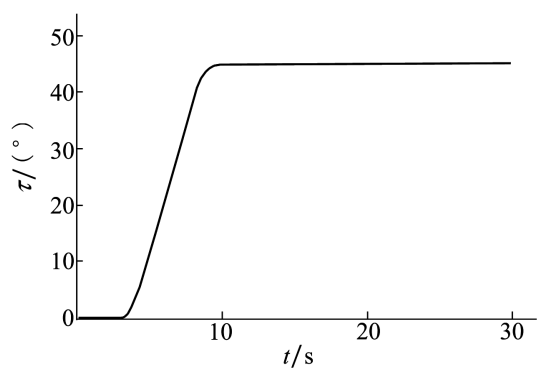

图 8 变构型下后掠角指令信号

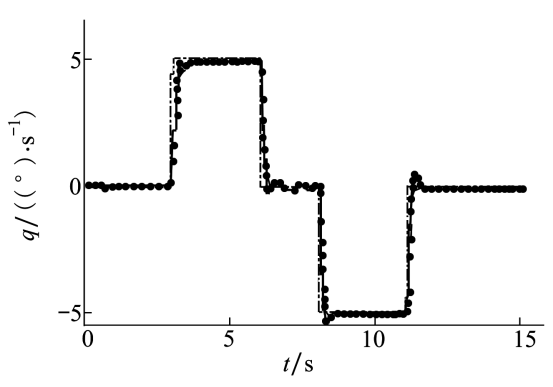

图 9 变构型下俯仰角速率变化曲线 


$$
\frac{\tau_{d}}{\tau_{d 0}}=\frac{5}{s^{2}+4 s+5}
$$

变体飞机后掠角发生变化导致整个模型发生改 变,可以将其看做被控对象的干扰项,在变体飞机变 化过程中对其进行控制考验了控制算法的鲁棒性和 自适应能力。通过图 9 可以看出基于 RBFNN 的鲁 棒自适应算法能更加快速地响应模型变化过程的控 制问题。传统反步法和本文提出方法的俯仰角速率 跟踪误差均方根分别是 0.7334 和 0.7058 , 相比之 下传统的反步法误差要高出 $3.9104 \%$ 。

从控制器最终输出结果看, 在 $8.1 \mathrm{~s}$ 时, 2 种方 法的出舵量出现最大偏差, 本文方法计算的升降舵 出舵量高出传统反步法的 $10.36 \%$, 印证了上文所得 出的结论。

\section{3 姿态环仿真验证}

上一节给出了本文提出方法在角速率环下的仿 真测试, 但是整体算法的优越性并没有完全体现出 来。俯仰角速率信号属于飞机控制系统中的快变 量, 对于系统的响应比较快, 而 RBFNN 在线逼近系 统的不确定项会需要一定的时间, 所以在内环控制 上, 该方法的优势并没有完全体现出来, 所以需要在 姿态环中进行仿真验证。

\section{1) 固定构型仿真对比}

固定后掠角构型为 $0^{\circ}\left(\tau=0^{\circ}\right)$, 选择迎角增量 $\Delta \alpha_{\text {cmd }}=1.833^{\circ}$ 为参考指令。初始飞行状态设为 $h=$ $1000 \mathrm{~m}, V=30 \mathrm{~m} / \mathrm{s}$, 迎角指令在 $2 \mathrm{~s}$ 后从初始状态 增加到 $5^{\circ}$ 。图 10 展示了仿真结果。

为了增加对比效果, 除了传统反步法和本文提 出方法设计控制器以外, 还设计对内环控制使用本 文方法, 外环使用传统反步法的控制器, 这 3 种控制 器对比结果如表 6 所示, 可以看出本文提出方法的 跟踪误差均方根最低, 传统反步法比其高出 $30.4149 \%$, 而且调节时间耗时最短, 比传统反步法 快 $61.82 \%$ 。

从控制器最终输出结果看, 2 种方法的出舵量 在控制指令给出后的 $0.12 \mathrm{~s}$ 内出舵偏差非常明显, 传统反步法的响应迟滞很明显, 动态性能欠佳。

表 6 固定构型下俯仰角跟踪误差的均方根对比

\begin{tabular}{cccc}
\hline 方法 & 均方根数值 & 对比 $/ \%$ & 调节时间 $/ \mathrm{s}$ \\
\hline 传统反步法 & 0.2670 & 0 & 1.10 \\
本文方法(内环) & 0.2493 & 7.0699 & 1.00 \\
本文方法(内外环) & 0.2047 & 30.4149 & 0.42 \\
\hline
\end{tabular}

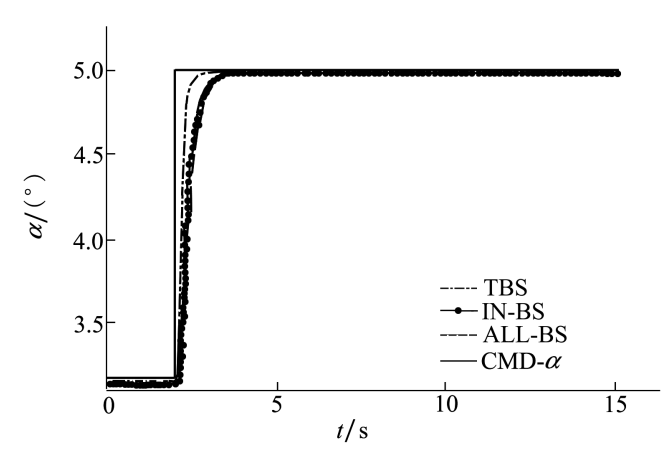

图 10 迎角变化曲线

2) 变构型仿真对比

为了进一步测试本文提出方法的性能, 将在给 定控制指令的同时对构型也进行改变。后掠角的变 化情况与 3.2 节的图 8 相同, 其余仿真条件与上小 节相同,其仿真结果如图 11 所示。

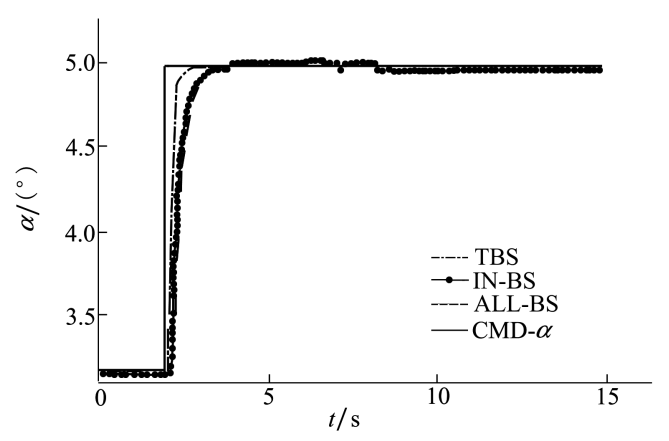

图 11 迎角变化曲线

与上一小节的结论相同, 后掠翼的变化会对控 制带来干扰, 使得控制的跟踪精度要低于固定构型, 但还是可以从仿真结果中看出基于 RBFNN 的鲁棒 自适应算法的优越性。从表 7 中可以看出在变构型 下本文提出方法跟踪误差还是最低, 传统反步法比 其高出 $30.7053 \%$, 而且调节时间耗时传统反步法 快 $61.82 \%$ 。

表 7 变构型下俯仰角跟踪误差的均方根对比

\begin{tabular}{cccc}
\hline 方法 & 均方根数值 & 对比 $/ \%$ & 调节时间 $/ \mathrm{s}$ \\
\hline 传统反步法 & 0.2673 & 0 & 1.10 \\
本文方法(内环) & 0.2495 & 7.1051 & 1.00 \\
本文方法(内外环) & 0.2045 & 30.7053 & 0.42 \\
\hline
\end{tabular}

从控制器最终输出结果看, 本文提出方法的出 舵量在控制指令给出后的 $0.12 \mathrm{~s}$ 内与传统反步法出 舵偏差非常明显，而这段时间也是后掠翼和控制指 
令同时作用的时间, 这是基于 RBFNN 的鲁棒自适 应算法对于这段过程额外的修正, 所以比传统反步 法有着更加优越的动态性能。

\section{4 结 论}

本文针对变体飞机非线性模型的不确定性问 题, 提出了一种基于 RBFNN 的鲁棒自适应方法, 经 过仿真验证表明该方法能很好地跟踪期望信号并能 保证控制的鲁棒性。通过自适应项和鲁棒项的引 人,该方法可以使变体飞机非线性模型对控制指令 的响应快速和精确。通过 Lyapunov 稳定性分析, 证 明该方法设计的控制律能够保证跟踪误差收敛且一 致有界, 且能有效抑制系统中不确定的影响。如何 根据实际需要选择整定自适应参数是下一步将要开 展的内容。

\section{附录 I 气动力和气动力矩方程}

$$
\begin{aligned}
& C_{\mathrm{D}}(\tau)=C_{\mathrm{D} 0}(\tau)+C_{\mathrm{D} d e}(\tau) \delta_{e} \\
& \left\{C_{\mathrm{L}}(\tau)=C_{\mathrm{L} 0}(\tau)+C_{\mathrm{L} q}(\tau) \cdot \frac{c_{A}(\tau)}{2 V} \cdot q+C_{\mathrm{L} d e}(\tau) \cdot \delta_{e}\right. \\
& C_{m}=C_{m 0}(\tau)+\frac{c_{A}(\tau)}{2 V} C_{m q}(\tau) \cdot q+C_{m d e}(\tau) \cdot \delta_{e} \\
& Q=\frac{1}{2} \rho V^{2}, \\
& \left\{\begin{array}{l}
L_{w}=C_{L}(\tau) \cdot Q \cdot S_{w}(\tau) \\
D_{w}=C_{D}(\tau) \cdot Q \cdot S_{w}(\tau) \\
Y_{w}=C_{Y}(\tau) \cdot Q \cdot S_{w}(\tau)
\end{array}\right. \\
& \left\{\begin{array}{l}
M l_{w}=C_{l}(\tau) \cdot Q \cdot S_{w}(\tau) \cdot b(\tau) \\
M m_{w}=C_{m}(\tau) \cdot Q \cdot S_{w}(\tau) \cdot c_{A}(\tau) \\
M n_{w}=C_{n}(\tau) \cdot Q \cdot S_{w}(\tau) \cdot b(\tau)
\end{array}\right. \\
& {\left[\begin{array}{l}
F_{b w x} \\
F_{b w y} \\
F_{b w z}
\end{array}\right]=\left[\begin{array}{ccc}
\cos \alpha \cos \beta & -\cos \alpha \sin \beta & -\sin \alpha \\
\sin \beta & \cos \beta & 0 \\
\sin \alpha \cos \beta & -\sin \alpha \sin \beta & \cos \alpha
\end{array}\right]\left[\begin{array}{c}
-D_{w} \\
Y_{w} \\
-L_{w}
\end{array}\right]}
\end{aligned}
$$

由于计算 CFD 数据时将气动力和力矩的参考 点固定在机体系中, 而变体飞机的重心是随着变体 飞机后掠角的改变而改变的, 由于力线平移定理 (theorem of parallel transfer the line of action of a force) 可知此变化对于气动力的没有影响, 但对于 飞机的气动力矩将产生巨大的影响, 所以要对飞机 的气动力矩进行修正。因此作用于飞行器上的总气 动力矩计算方法如下

$$
\left\{\begin{array}{l}
M_{b x}=M l_{w}-z_{c g} F_{b w y}+y_{c g} F_{b w z} \\
M_{b y}=M m_{w}-x_{c g} F_{b w z}+z_{c g} F_{b w x} \\
M_{b z}=M n_{w}+x_{c g} F_{b w y}+y_{c g} F_{b w x}
\end{array}\right.
$$

式中, $\left(x_{c g}, y_{c g}, z_{c g}\right)$ 是 $\mathrm{CFD}$ 计算参考原点与实际变 体飞机质心之间的距离。

\section{附录 II 文中涉及的变量}

$$
\left\{\begin{array}{l}
\dot{x_{1}}=f_{1}(\boldsymbol{x})+g_{1}(\boldsymbol{x}) x_{2}+\Delta_{1} \\
\dot{x_{2}}=f_{2}(\boldsymbol{x})+g_{2}(\boldsymbol{x}) u+\Delta_{2}
\end{array}\right.
$$

式中, $\boldsymbol{x}=[\alpha q V \gamma h]^{\mathrm{T}}, x_{1}=\alpha, x_{2}=q, u=\delta_{e}$ 。

$\left\{\begin{array}{l}f_{1}(\boldsymbol{x})=\frac{1}{m V \cos \beta}\left(-L_{w}+m g \cos \gamma \cos \mu-T \sin \alpha\right) \\ g_{1}(\boldsymbol{x})=1\end{array}\right.$ $\left\{\begin{array}{l}f_{2}(\boldsymbol{x})=\frac{Q S_{w} c_{A}}{I_{y}}\left(C_{m 0}+\frac{c_{A} C_{m q} q}{2 V}\right)+\frac{Q S_{w} x_{c g}}{I_{y}} C_{D 0} \sin \alpha+ \\ \frac{Q S_{w} x_{c g}}{I_{y}}\left(C_{L 0}+\frac{c_{A} C_{L q} q}{2 V}\right) \cos \alpha \\ g_{2}(\boldsymbol{x})=\frac{Q S_{w}}{I_{y}}\left(x_{c g}\left(C_{L \delta e} \cos \alpha+C_{D \delta e} \sin \alpha\right)+c_{A} C_{m \delta e}\right)\end{array}\right.$ 


\section{参考文献:}

[1] SHI R, PENG J. Morphing Strategy Design for Variable-Wing Aircraft [ C] // 15th AIAA Aviation Technology, Integration, and Operations Conference. 2015: 3002

[2] WU Z, LU J, RAJPUT J, et al. Adaptive Neural Control Based on High Order Integral Chained Differentiator for Morphing Aircraft [EB/OL] . (2015-10-25) [2019-08-29]. https://www.hindawi.com/journals/mpe/2015/787931/

[3] SEIGLER T M, NEAL D A, BAE J S, et al. Modeling and Flight Control of Large-Scale Morphing Aircraft[J]. Journal of Aircraft, $2007,44(4): 1077-1087$

[4] WU Z, LU J, ZHOU Q, et al. Modified Adaptive Neural Dynamic Surface Control for Morphing Aircraft with Input and Output Constraints [J]. Nonlinear Dynamics, 2017, 87(4) : 2367-2383

[5] SHI R, WAN W. Analysis of Flight Dynamics for Large-Scale Morphing Aircraft[J]. Aircraft Engineering and Aerospace Technology: An International Journal, 2015, 87(1) : 38-44

[6] O'GRADY B. Multi-Objective Optimization of a Three Cell Morphing Wing Substructure[D]. Dayton: University of Dayton, 2010

[7] JOHNSON T, FRECKER M, ABDALLA M, et al. Nonlinear Analysis and Optimization of Diamond Cell Morphing Wings [J]. Journal of Intelligent Material Systems and Structures, 2009, 20(7) : 815-824

[8] JOO J J, SANDERS B. Optimal Location Of Distributed Actuators Within an In-Plane Multi-Cell Morphing Mechanism[J]. Journal of Intelligent Material Systems and Structures, 2009, 20(4) : 481-492

[9] ANDERSEN G, COWAN D, PIATAK D. Aeroelastic Modeling, Analysis and Testing of a Morphing Wing Structure[C] // 48th AIAA/ASME/ASCE/AHS/ASC Structures, Structural Dynamics, and Materials Conference, 2007: 1734

[10] SEIGLER T, NEAL D, INMAN D. Dynamic Modeling of Large-Scale Morphing Aircraft[C]//47th AIAA/ASME/ASCE/AHS/ ASC Structures, Structural Dynamics, and Materials Conference, 2006: 1893

[11] OBRAdoviC B, SUBBARAO K. Modeling of Flight Dynamics of Morphing Wing Aircraft [ J ]. Journal of Aircraft, 2011 , 48 (2) : 391-402

[12] YUE T, WANG L, AI J. Longitudinal Linear Parameter Varying Modeling and Simulation of Morphing Aircraft [ J]. Journal of Aircraft, 2013, 50(6): 1673-1681

[13] BOOTHE K, FITZPATRICK K, LIND R. Controllers for Disturbance Rejection for A Linear Input-Varying Class of Morphing Aircraft $[\mathrm{C}] / /$ 46th AIAA/ASME/ASCE/AHS/ASC Structures, Structural Dynamics and Materials Conference, 2005 : 2374

[14] BEAVERSTOCK C S, AJAJ R, FRISWELL M I, et al. Effect of Span-Morphing on the Flight Modes, Stability \& Control[ C] // AIAA Guidance, Navigation, and Control (GNC) Conference, 2013: 4993

[15] YUE T, WANG L, AI J. Gain Self-Scheduled $H_{\infty}$ Control for Morphing Aircraft in the Wing Transition Process Based on an LPV $\operatorname{Model}[\mathrm{J}]$. Chinese Journal of Aeronautics, 2013, 26(4) : 909-917

[16] BALDELLI D H, LEE D H, SÁNCHEZ PEÑA R S, et al. Modeling and Control of an Aeroelastic Morphing Vehicle[J]. Journal of Guidance, Control, and Dynamics, 2008, 31(6) : 1687-1699

[17] GANDHI N, JHA A, MONACO J, et al. Intelligent Control of a Morphing Aircraft [ C] // 48th AIAA/ASME/ASCE/AHS/ASC Structures, Structural Dynamics, and Materials Conference, 2007: 1716

[18] NOBLEHEART W, SHIVANAPURA LAKSHMIKANTH G, CHAKRAVARTHY A, et al. Single Network Adaptive Critic (SNAC) Architecture for Optimal Tracking Control of a Morphing Aircraft during a Pull-up Maneuver [C] // AIAA Guidance, Navigation, and Control Conference, 2013: 5003

[19] VALASEK J, LAMPTON A, MARWAHA M. Morphing Unmanned Air Vehicle Intelligent Shape and Flight Control[EB/OL]. (2009-04-15 ) [2019-08-29]. https : // www. researchgate.net/publication/228644798_Morphing_Unmanned_Air_Vehicle_Intelligent_Shape_and_Flight_control

[20] CHENLIANG W, YAN L. Adaptive Dynamic Surface Control for Linear Multivariable Systems[J]. Automatica, 2010, 46( 10) : 1703-1711

[21] LIU Y J, CHEN C L P, WEN G X, et al. Adaptive Neural Output Feedback Tracking Control for a Class of Uncertain DiscreteTime Nonlinear Systems[J]. IEEE Trans on Neural Networks, 2011, 22(7) : 1162-1167 
[22] CHEN M, TAO G, JIANG B. Dynamic Surface Control Using Neural Networks for a Class of Uncertain Nonlinear Systems with Input Saturation[J]. IEEE Trans on Neural Networks and Learning Systems, 2014, 26(9) : 2086-2097

[23] CAO C, HOVAKIMYAN N. Novel L1 Neural Network Adaptive Control Architecture with Guaranteed Transient Performance[J]. IEEE Trans on Neural Networks, 2007, 18(4) : 1160-1171

\title{
A High Precision Adaptive Back-Stepping Control Method for Morphing Aircraft Based on RBFNN Method
}

\author{
QIAO Fuxiang, SHI Jingping, ZHANG Weiguo, LYU Yongxi, QU Xiaobo \\ (School of Automation, Northwestern Polytechnical University, Xi'an 710072, China)
}

\begin{abstract}
To overcome the uncertainties of the nonlinear model of a morphing aircraft, this paper presents a highprecision adaptive back-stepping control method based on the radial basis function neural network (RBFNN). Firstly, based on the analysis of static and dynamic aerodynamic parameters of the morphing aircraft, its nonlinear control law is designed by using the conventional back-stepping method. The RBFNN is introduced to approximate online the uncertain terms of the nonlinear control law so as to improve its robustness. The robust term is designed to eliminate the approximation error caused by the RBFNN. Secondly, the tracking differentiator is designed through solving the virtual control variables, thus solving the "differential expansion" problem existing in the traditional back-stepping method. The Lyapunov stability analysis proves that our method can ensure that the tracking error of a closed-loop system converges finally and that its signals are uniformly bounded. Finally, the digital simulation model of the morphing aircraft is established with the MATLAB/Simulink; our method is compared with the conventional back-stepping control method. The simulation results show that our method has a higher control precision and stronger robustness.
\end{abstract}

Keywords : morphing aircraft; back-stepping control; radial basis function neural network ( RBFNN ); adaptive control 